\title{
Erratum to: Comparative QSRR Modeling of Nitrobenzene Derivatives Based on Original Molecular Descriptors and Multivariate Image Analysis Descriptors
}

\author{
Zahra Garkani-Nejad • Mohammad Ahmadvand
}

Published online: 12 July 2011

(C) Springer-Verlag 2011

Erratum to: Chromatographia (2011) 73:733-742

DOI 10.1007/s10337-011-1969-7

In the original article, a novel approach was used to compare different models based on the sum of ranking differences (SRD). We have ranked the retention data properly but used retention data instead of rank numbers. Thus, the ordering remained correct, the values obtained for the different models are not comparable with other ordering schemes. These values are shown in Table 4 . Now, SRD values of Table 4 should read: $M L R=50$, $\mathrm{PLS}=52, \quad \mathrm{ANN}-\mathrm{LM}=50, \mathrm{CR}-\mathrm{PCR}=128, \mathrm{CR}-\mathrm{PC}-$ $\mathrm{ANN}-\mathrm{LM}=40$, using row average of retention times as reference. However, our conclusion remains. Based on the SRD values, the CR-PC-ANN-LM model with the minimum SRD value is the best one.

The online version of the original article can be found under doi:10.1007/s10337-011-1969-7.

Z. Garkani-Nejad ( $₫) \cdot$ M. Ahmadvand

Chemistry Department, Faculty of Science,

Vali-e-Asr University, Rafsanjan, Iran

e-mail: garakani@vru.ac.ir 